CHAPTER

\title{
Isolation,
}

cryotomography, and

three-dimensional

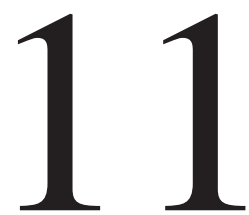

\section{reconstruction of centrioles}

Paul Guichard, Virginie Hamel, Aitana Neves, Pierre Gönczy ${ }^{1}$ Swiss Institute for Experimental Cancer Research (ISREC), School of Life Sciences, Swiss Federal Institute of Technology (EPFL), Lausanne, Switzerland

${ }^{1}$ Corresponding author: E-mail: pierre.gonczy@epfl.ch

\section{CHAPTER OUTLINE}

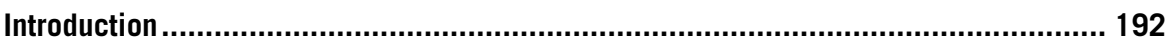

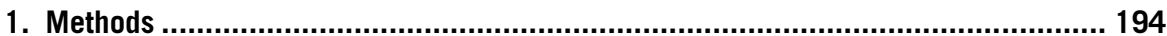

1.1 Materials and Reagents .......................................................... 194

1.1.1 Trichonympha and centriole isolation ........................................ 194

1.1.2 Detection of Trichonympha centrioles by immunofluorescence........... 195

1.1.3 Electron microscopy .................................................................... 195

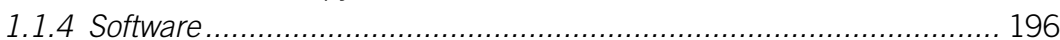

1.2 Trichonympha Centriole Isolation ................................................ 196

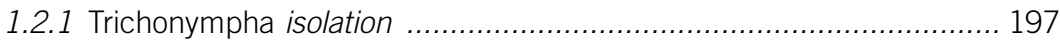

1.2.2 Isolation of centrioles from Trichonympha.................................... 197

1.3 Analysis of Isolated Trichonympha Centrioles .................................. 197

1.3.1 Immunofluorescence experiment ................................................ 199

1.3.2 Analysis by transmission electron microscopy ................................ 200

1.4 Cryotomography of Centrioles .................................................. 200

1.4.1 Sample preparation and transfer into a cryomicroscope................... 200

1.4.2 Tomogram acquisition ............................................................... 202

1.4.3 Tomogram reconstruction using IMOD ......................................... 203

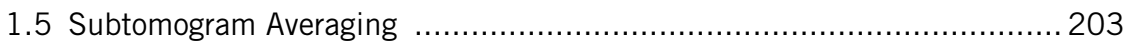

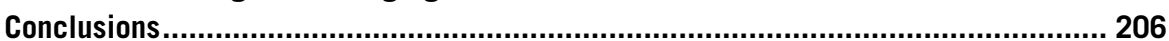

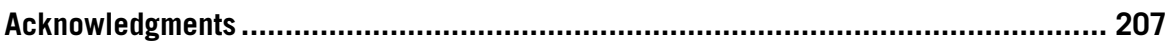

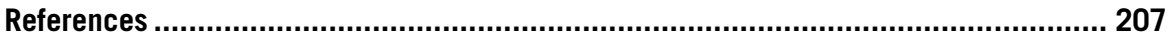




\section{Abstract}

Centrioles and basal bodies (referred to hereafter as centrioles for simplicity) are microtubule-based cylindrical organelles that are typically $\sim 450-\mathrm{nm}$ long and $\sim 250 \mathrm{~nm}$ in diameter. The centriole is composed of three distinct regions: the distal part characterized by microtubule doublets, the central core that harbors microtubule triplets, which are also present in the proximal part that also contains the cartwheel, a structure crucial for centriole assembly. The cartwheel was initially revealed by conventional electron microscopy of resin-embedded samples and is thought to impart the near universal ninefold symmetry of centrioles. Deciphering the native architecture of the cartwheel has proven challenging owing to its small dimensions and the difficulties in isolating it. Here, we present a method to purify and analyze the structure of the exceptionally long Trichonympha centriole by cryotomography and subtomogram averaging. Using this method, we revealed the native architecture of the proximal cartwheel-containing region at $\sim 40 \AA$ A-resolution. This method can be applied as a general strategy for uncovering the structure of centrioles in other species.

\section{INTRODUCTION}

Centrioles are among the largest macromolecular complexes of eukaryotic cells and exhibit a remarkably conserved cylindrical structure containing nine microtubule blades (term used from here on to collectively refer to microtubule doublets and triplets). The correct assembly of centrioles is critical for the formation of centrosomes and cilia (reviewed in Nigg \& Raff (2009)). Deciphering the architecture of centrioles is key for understanding overall centriole assembly and function (reviewed in Gönczy (2012)). Due to the typically small dimensions of centrioles ( 450-nm long and $\sim 250$-nm diameter), electron microscopy of resin-embedded samples has been the method of choice over the last 60 years to study their ultrastructure (e.g., Allen, 1969; Bornens, Paintrand, Berges, Marty, \& Karsenti, 1987; Cavalier-Smith, 1974; Dippell, 1968; Geimer \& Melkonian, 2004; Vorobjev \& Chentsov, 1982). These studies showed that the canonical centriole is not only composed of nine microtubule blades, but also displays other signature features. Among these are the distal and subdistal appendages, which are needed for anchorage to the plasma membrane during cilium formation. Another example is the central core that comprises periodic densities along the A-microtubule. Finally, the proximal region contains a remarkable structure called the cartwheel that is thought to impart the ninefold symmetry of the entire centriole (Figure 1(A)).

Despite these advances, analysis of resin-embedded specimens is limited by the steps necessary for preparation of samples for electron microscopy. The successive steps of chemical/cryofixation, dehydration in pure ethanol, as well as staining using heavy metals such as osmium tetroxide and uranyl acetate, induce artifacts of sample preparation and reduce overall resolution (Dubochet \& Sartori Blanc, 2001). These limitations have been overcome by the development over the last 30 years of cryoelectron microscopy, which allows observation of biological samples in a hydrated 
(A)

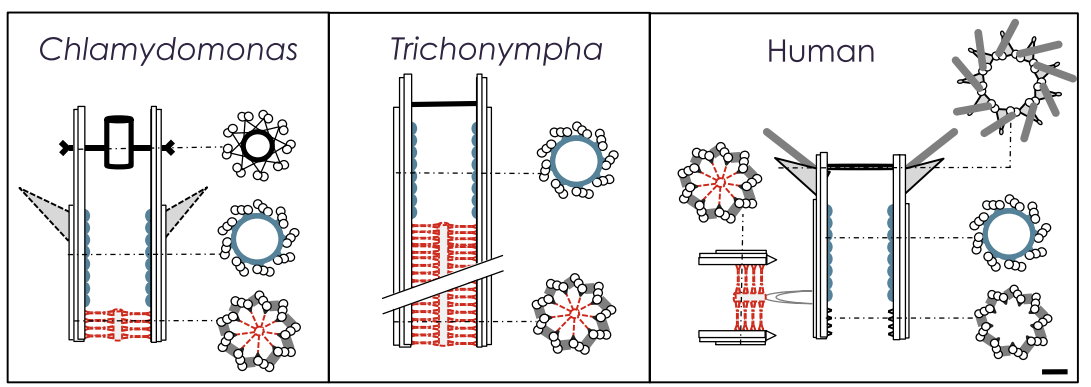

(B)

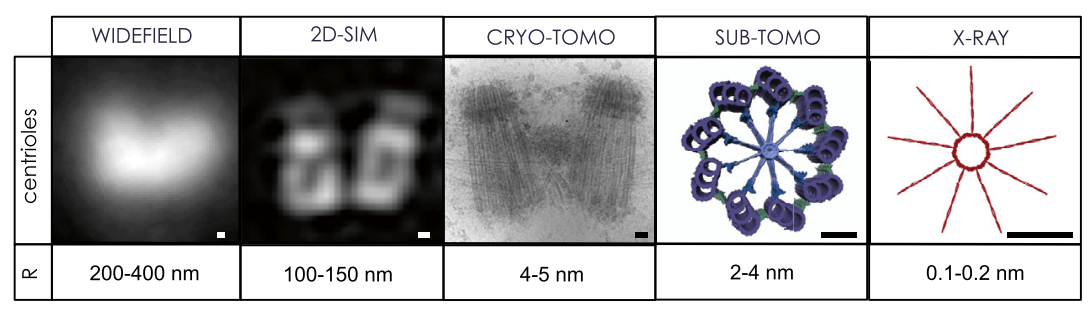

(C)

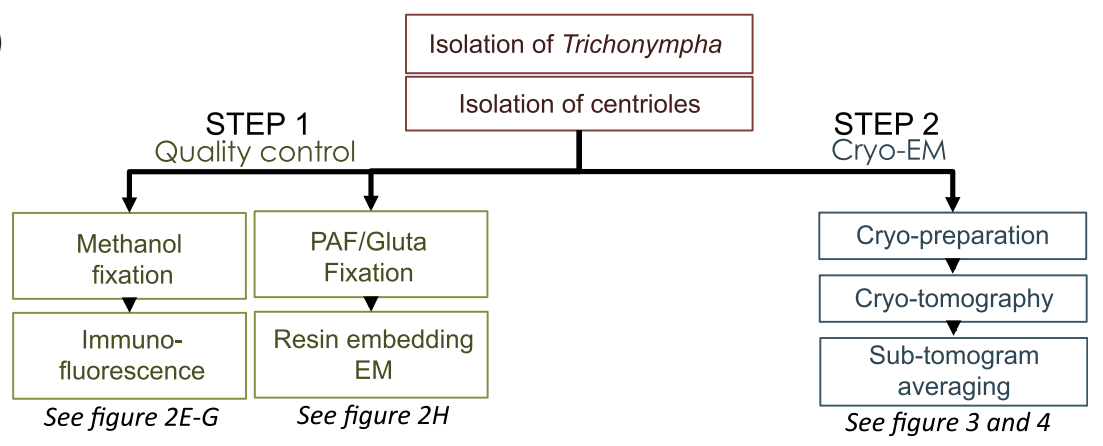

FIGURE 1 Analysis of centriolar architecture.

\begin{abstract}
(A) Schematic representation of centriolar architecture in Chlamydomonas, Trichonympha, and human cells. Note that the overall organization of the centriole is conserved, with the ninefold symmetrical arrangement of outer microtubule blades (small circles), the central core (blue (dark gray in print versions)), and the cartwheel (red (gray in print versions)). (B) Methods utilized to analyze centriolar structure at different resolutions (R). (C) Summary of the steps taken to perform cryomicroscopy analysis of purified Trichonympha centrioles. Scale bar: $50 \mathrm{~nm}$.
\end{abstract}

state, also dubbed native state. This method has been developed by the group of Jacques Dubochet and relies on the ability to create a state close to liquid water but frozen at $-180{ }^{\circ} \mathrm{C}$, the vitreous state (Dubochet, 2012; Dubochet \& McDowall, 1981). Briefly, samples such as proteins, with an overall size not exceeding several hundreds of nanometers, are deposited directly on EM grids and quickly plunged in liquid ethane so as to trap them in this vitreous state prior to analysis by 
cryomicroscopy. In this condition, the sample does not need to be stained, as the inherent electron density of the sample generates the contrast on the image (Bouchet-Marquis \& Hoenger, 2011).

Over the following decades, important developments in image processing and especially in tomography and particle averaging have brought forward electron microscopy as an alternative method to analyze protein complexes, filling the gap between superresolution fluorescent microscopy techniques and atomic resolution methods such as X-ray crystallography (Figure 1(B)). With these advances, one can now thoroughly analyze the native structure of centrioles by cryomicroscopy using cryotomography and image processing (Guichard, Chrétien, Marco, \& Tassin, 2010; Guichard et al., 2012, 2013; Li, Fernandez, Marshall, \& Agard, 2011).

Here, we report a detailed protocol to perform isolation, cryotomography, and three-dimensional reconstruction of centrioles. To resolve the native architecture of the centriolar proximal region at $\sim 40$ - $\AA$ resolution, we take advantage of the centrioles found in the protist Trichonympha, which have a $\sim 40$ times longer proximal region than that of canonical centrioles, and exemplify a procedure that can be applied also to the study of centrioles from other species.

\section{METHODS}

\subsection{MATERIALS AND REAGENTS}

Listed below are specific material and reagents needed for the different steps of centriole analysis. Usual material for molecular biology is also needed (pipettes, tips, distilled water, etc.).

\subsubsection{Trichonympha and centriole isolation}

- Zootermopsis nevadensis termites

- Tweezer DUMONT n ${ }^{\circ} 5$ (Dumont, ref 0101-5-PO)

- Ice bucket

- 10-cm-diameter petri dish

- K-PIPES $10 \mathrm{mM}$ pH 7.2

- Differential interference contrast (DIC) microscope with a $20 \times$ objective

- Lysis buffer: 10-mM K-PIPES pH 7.2 + 0.5\% NP-40 (IGEPAL) + protease inhibitors Complete (SIGMA; 1/1000)

- Tabletop centrifuge at $4{ }^{\circ} \mathrm{C}$ or in a cold room

- 12-mm round coverslip

- Glass slide

- 15-ml Kontes ${ }^{\mathrm{TM}} \mathrm{KIMAX}^{\mathrm{TM}}$ Reusable high strength centrifuge tubes without cap (Fisher Scientific, ref 09-500-34) - "Corex" tubes

- 16-mm-diameter adapter rubber to put the Corex tubes in the rotor (Fisher Scientific, ref 05-569-11)

- JS-13 (Avanti) or HB6 (Beckman) centrifuge rotors (to be used for the Corex tubes) 
- Adapters for coverslips

- Curved-tip needle

- Sucrose

\subsubsection{Detection of Trichonympha centrioles by immunofluorescence}

- Methanol (100\%)

- Ceramic rack (Thomas Scientific, ref 8542E40)

- 6-well plate (used in cell culture)

- Parafilm

- Mouse monoclonal antiacetylated tubulin 6-11B-1 (Sigma-Aldrich, ref: T6793)

- Mouse centrin-2 antibody 20H5 (Merck Millipore, ref 04-1624)

- Secondary antibodies anti-mouse Alexa 568 (Life technologies, ref A11004)

- Secondary antibodies anti-rabbit Alexa 488 (Life technologies, ref A11034)

- PBS, Tween, BSA (PBT): Phosphate Buffered Saline (PBS) + Tween 0.05\% + Bovine Serum Albumin (BSA) 1\%

- Mounting medium: 4\% n-Propyl-Gallate; $1 \times$ PBS; $90 \%$ glycerol

- Nail polish (transparent)

- Fluorescence microscope (epifluorescence, confocal, $63 \times$ oil objective, etc.)

\subsubsection{Electron microscopy}

1.1.3.1 Conventional electron microscopy

- Nunc $^{\mathrm{TM}}$ Thermanox ${ }^{\mathrm{TM}}$ 13-mm-diameter coverslips (ThermoScientific, ref 174950)

- Paraformaldehyde (EMS, ref 15700)

- Glutaraldehyde (EMS, ref 16000)

- Fixative solution (PBS with 2\% paraformaldehyde and $1.5 \%$ glutaraldehyde)

- Cacodylate buffer 0.1 M, pH 7.4 (EMS, ref 11650)

- Osmium and Uranyl acetate (EMS, ref 19100 and ref 22400, respectively)

- Graded ethanol series: 50\%, 70\%, 90\%, 95\%, and 100\%

- Watch glass

- Durcupan resin (EMS, ref 14040)

- Vacuum oven (EMS, ref 3606)

- Razor blade

- Ultramicrotome for electron microscopy preparation

- Electron microscope operating at $80 \mathrm{KeV}$ for observation of resin section

\subsubsection{Cryomicroscopy}

- Tweezer Style L5 with clamping rings (EMS, ref 0508-L4-PO)

- 10-nm gold beads (Aurion, ref 210.133)

- Cryo-EM grids Lacey 300 mesh (EMS, ref LC325-Cu)

- Homemade cryoplunger or FEI Vitrobot or Leica EM GP system

- Liquid nitrogen

- Bottle of ethane gas

- Whatman filter paper $n^{\circ} 4$

- Cryomicroscope equipped with field emission gun (FEG) operating at $200 \mathrm{KeV}$ 


\subsubsection{Software}

\subsubsection{Tomogram acquisition}

- FEI tomogram acquisition

- JEOL tomogram acquisition

- SerialEM http://bio3d.colorado.edu/SerialEM/

\subsubsection{Tomogram reconstruction}

- Input/ouput plug-in to convert stack in an .mrc format: http://u759.curie.fr/en/ download/softwares/input-output/input-output-00741

- IMOD (etomo): http://bio3d.colorado.edu/imod/

\subsubsection{Image processing and subtomogram averaging}

- Matlab: mathworks.com/products/matlab/

- Coordinates interpolation: http://gonczy-lab.epfl.ch/resources

- Bsoft: http://lsbr.niams.nih.gov/bsoft/

- Spider: http://spider.wadsworth.org/spider_doc/spider/docs/spider.html

- PEET: http://bio3d.colorado.edu/PEET/

- Dynamo: https://dynamo.bioz.unibas.ch/

- Jsubtomo: http://www.opic.ox.ac.uk/wiki/index.php/Jsubtomo

Other software for tomography and image processing can be found at:

http://en.wikibooks.org/wiki/Software_Tools_For_Molecular_Microscopy/Specific_ packages.

\subsubsection{Visualization}

- ImageJ: http://imagej.nih.gov/ij/

- UCSF Chimera: http://www.cgl.ucsf.edu/chimera/

\subsection{TRICHONYMPHA CENTRIOLE ISOLATION}

The parabasalid protists Trichonympha are symbiotes of termites that live in their gut and break down the cellulose of the wood ingested by their host (Schmidt, 1956). To obtain a large amount of starting material, we used Zootermopsis nevadensis termites, in whose gut reside three species of Trichonympha: Trichonympha campanula, Trichonympha collaris, and Trichonympha sphaerica. Trichonympha campanula is the most abundant among these, such that the resulting isolated centrioles will be mostly from this species.

Note: After purification of the centrioles, we recommend not to freeze the sample because the slow formation of ice can affect sample integrity. In the following protocol, analyze the sample directly by immunofluorescence and conventional EM and in a second step, repeat the exact same protocol and directly perform cryo-EM (Figure 1(C)).

If the sample needs to be frozen, add sucrose with a final concentration of at least $30 \%$ and snap freeze the sample. In this condition, the sample can be still be 
analyzed by immunofluorescence and regular EM but not by cryo-EM due to the sucrose that prevents formation of good vitreous ice, thus leading to considerably reduced contrast (Grassucci, Taylor, \& Frank, 2007).

\subsubsection{Trichonympha isolation (Figure 2(A)-(B))}

- Place a petri dish on ice.

- Collect one Zootermopsis nevadensis termite and put it in the petri dish.

- Once the termite is anesthetized by cold (around $5 \mathrm{~min}$ ), remove its head with tweezers (Figure 2(A), steps 1 and 2).

- Pull the gut out from the termite's abdomen with the tweezers. The gut comes out easily but be gentle so as not to break it (Figure 2(A), steps 3 and 4).

- Tear the gut open using the tweezers as much as possible.

- Place the gut in an Eppendorf tube containing $1 \mathrm{~mL}$ of 10-mM K-PIPES pH 7.2. The gut contains a mix of bacteria and different types of flagellates, including Trichonympha (Figure 2(A), step 5).

- Let Trichonympha cells sediment for $10 \mathrm{~min}$ on ice; the bacteria and smaller protists will remain in the supernatant (Figure 2(B)).

- Carefully aspirate the supernatant with a pipette and then resuspend the sedimented material in 10-mM K-PIPES pH 7.2 (Figure 2(B)). Let the Trichonympha cells sediment for $10 \mathrm{~min}$ and repeat the entire procedure (sedimentation, removal of the supernatant, and resuspension of the pellet) 3 times.

- Visualize the integrity of the cells by DIC microscopy and verify that most bacteria are gone (Figure 2(C)).

\subsubsection{Isolation of centrioles from Trichonympha}

All steps hereafter should be performed at $4{ }^{\circ} \mathrm{C}$.

- Lyse the cells by adding $1 \mathrm{~mL}$ of lysis buffer to the sedimented Trichonympha cells.

- Incubate $20 \mathrm{~min}$ (if you want to keep the rostrum) or $1 \mathrm{~h}$ (if you want to dissociate the rostrum) (Figure 2(D)). Most centriole-flagella are released after 20 min.

- Centrifuge at $500 \mathrm{~g}$ for $3 \mathrm{~min}$ in a tabletop centrifuge to remove the cellular debris, collect the supernatant, and centrifuge it at $1000 \mathrm{~g}$ for $5 \mathrm{~min}$. The pellet contains isolated centrioles still attached to their flagella (Figure 2(E)).

\subsection{ANALYSIS OF ISOLATED TRICHONYMPHA CENTRIOLES (FIGURE 2(E-H))}

To determine the quality of the sample, an immunofluorescence experiment and an analysis using conventional transmission electron microscopy are performed (Figure 2(E)-(H)). The first method serves to evaluate whether select proteins have remained in place during isolation for instance by using antibodies that recognize centriolar proteins. The second method evaluates the purity of the sample and its structural integrity. 
198 CHAPTER 11 Isolation, cryotomography, and 3D reconstruction of centrioles
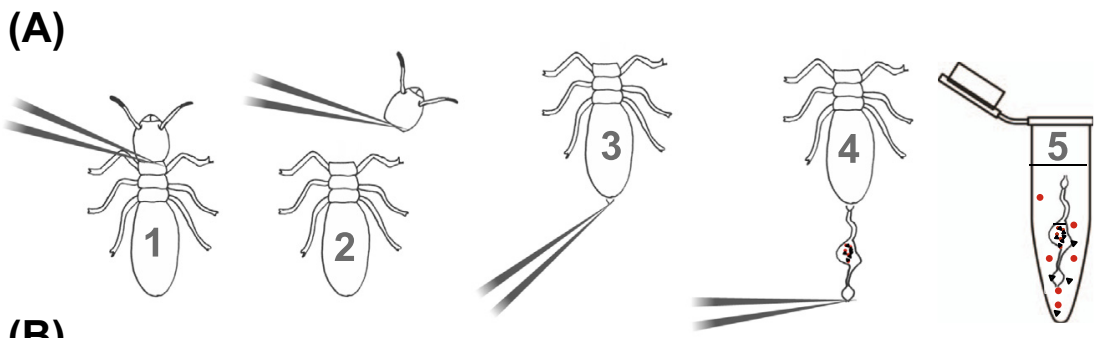

(B)
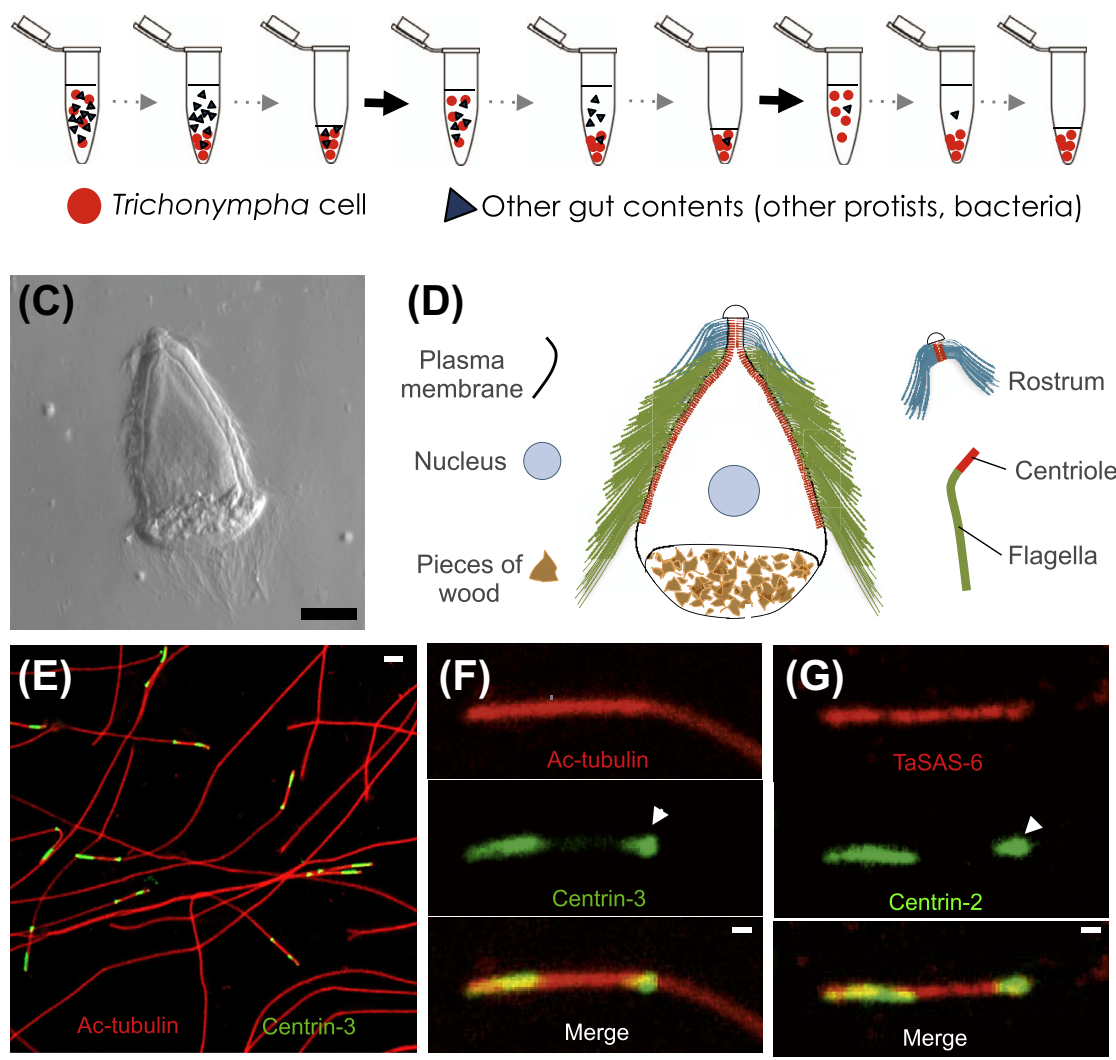

(H)

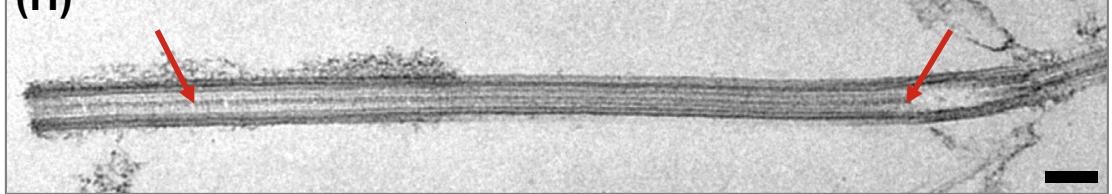

FIGURE 2 Isolation of Trichonympha centrioles.

(A) Dissection of Zootermopsis nevadensis termite and extraction of its gut. (B) Trichonympha cells are isolated from the rest of the gut contents by three rounds of washes 


\subsubsection{Immunofluorescence experiment}

- Place an adapter inside a 15-mLCorex tube containing $5 \mathrm{~mL}$ of 10-mM K-PIPES buffer and insert a 12-mm diameter round glass coverslip (For details, see chapter on purification of centrosomes from mammalian cell lines).

- Load $50 \mu \mathrm{L}$ of isolated centrioles preparation into the K-PIPES buffer solution in the Corex tube.

- Centrifuge at $11,000 \mathrm{~g}$ for $10 \mathrm{~min}$ at $4{ }^{\circ} \mathrm{C}$ using a JS-13 (Avanti) or HB6 (Beckman) swinging rotor with the corresponding rubber adapter.

- Gently pull out the adapter from the Corex tube using a curved-tip needle and carefully remove the coverslip with tweezers (For details, see chapter on purification of centrosomes from mammalian cell lines).

- Fix the coverslip by putting it for $4 \mathrm{~min}$ in precooled pure methanol at $-20{ }^{\circ} \mathrm{C}$ in a ceramic rack.

- Place the coverslip in a well of a 6-well plate filled with PBS and rinse for $5 \mathrm{~min}$.

- Centrioles are subsequently stained with antibodies against acetylated tubulin (1/1000 dilution in PBT) to mark centriolar microtubules and flagella, as well as centrin-2 (1/1000 dilution in PBT) to mark only centrioles. Practically speaking, the coverslip is deposited on a strip of Parafilm and covered by $50-100 \mu \mathrm{L}$ of antibody, incubating for $1 \mathrm{~h}$ at room temperature with a lid to prevent dehydration.

- Wash the coverslip by putting it in a well of a 6-well plate filled with PBS for $5 \mathrm{~min}$.

- Incubate with secondary antibodies diluted in PBT (anti-mouse Alexa-568 1/1000 and anti-rabbit Alexa 488 1/1000) for $45 \mathrm{~min}$ at room temperature as for the primary antibodies.

- Wash the coverslip in PBS for 5 min.

- Mount the coverslip on a glass slide. Pipette $6 \mu \mathrm{L}$ of mounting medium onto a glass slide. Take the coverslip with tweezers to invert it (sample facing down) and gently deposit it onto the drop of mounting medium. Seal the coverslip with nail polish.

and sedimentation. (C) Intact isolated Trichonympha cells under DIC microscope. Scale bar: $20 \mu \mathrm{m}$. (D) Schematic representation of a Trichonympha cell. Note the rostrum, which dissociates later than the cell body. (E-G) Isolated Trichonympha centrioles connected to flagella stained with antibodies against centrin (green) $(E-G)$ and acetylated tubulin (red, E, F) or TaSAS-6 (red, G). Note that in the Trichonympha centriole, the pattern of centrin (centrin-2 or centrin-3) staining is different from that observed for Chlamydomonas or human centrioles. Indeed, beside the evolutionary conserved localization of the proteins at the distal region of centrioles (arrowheads, $F, G$ ), centrin antibodies also decorate the proximal-most third of the proximal region. Scale bars represent $2 \mu \mathrm{m}$ in $(E)$ and $500 \mathrm{~nm}$ in $(F-G)$. (H) Conventional transmission electron microscopy of the exceptionally long Trichonympha centriole. Note that the central hub of the cartwheel is visible along the centriole (red arrow). Scale bar: $200 \mathrm{~nm}$. (See color plate) 
- Analyze the stained centrioles under an epifluorescence microscope or a confocal microscope as shown in Figure 2(E)- $(\mathrm{G})$.

In addition to this analysis by fluorescent microscopy, the structural integrity of isolated centrioles and flagella is analyzed by conventional electron microscopy (Figure 2(H)). To do so:

\subsubsection{Analysis by transmission electron microscopy}

- Place an adapter inside a 15-mL Corex tube containing $5 \mathrm{~mL}$ of 10-mM K-PIPES buffer and insert a 13-mm diameter Thermanox coverslip (ThermoScientific).

- Load $50 \mu \mathrm{L}$ of isolated centrioles directly into the K-PIPES buffer in the Corex tube.

- Centrifuge at $11,000 \mathrm{~g}$ for $10 \mathrm{~min}$ at $4{ }^{\circ} \mathrm{C}$ using a JS-13 (Avanti) or HB6 (Beckman) swing rotor with the corresponding rubber adapter.

- Gently pull out the adapter from the Corex tube using a curved-tip needle and carefully remove the coverslip with tweezers. Note that the following steps are conducted at room temperature.

- Fix the coverslip by putting it in a well of a 6 -well plate containing $2 \mathrm{~mL}$ of fixative solution (PBS with 2\% paraformaldehyde and $1.5 \%$ glutaraldehyde) for $60 \mathrm{~min}$.

- Wash the coverslips 3 times in cacodylate buffer $(0.1 \mathrm{M}, \mathrm{pH} 7.4)$ for $5 \mathrm{~min}$.

- Postfix with $1 \%$ osmium tetroxide in cacodylate buffer for $60 \mathrm{~min}$.

- Wash coverslip with distilled water (2 times for $5 \mathrm{~min}$ ).

- Stain with $2 \%$ uranyl acetate for $30 \mathrm{~min}$.

- Wash once coverslips in distilled water.

- In watch glasses, dehydrate in a graded alcohol series, 3 min each $(2 \times 50 \%$, $1 \times 70 \%, 1 \times 90 \%, 1 \times 95 \%, 2 \times 100 \%$ ).

- Add 1:1 EtOH (100\%): Durcupan for $30 \mathrm{~min}$.

- Embed in $100 \%$ Durcupan for at least $4 \mathrm{~h}$ at room temperature.

- Incubate in oven at $65^{\circ} \mathrm{C}$ overnight.

- Separate the Thermanox coverslip from the resin using a razor blade.

- Perform serial 60-nm thick sections using an ultramicrotome.

Note: By keeping the resin surface parallel to the knife in the ultramicrotome, the centrioles are cut longitudinally, thus allowing observation of the structure through its entire length (Figure 2(H)).

\subsection{CRYOTOMOGRAPHY OF CENTRIOLES (FIGURE 3)}

Once the centriole isolation procedure is robust as judged by immunofluorescence and electron microscopy, proceed to repeat the centriole isolation procedure and perform analysis by cryomicroscopy (Figure 3).

\subsubsection{Sample preparation and transfer into a cryomicroscope}

- Mix the sample with 10-nm gold beads, so as to reach a concentration of at least 10 gold beads per field of view in cryo-EM at the magnification used for 

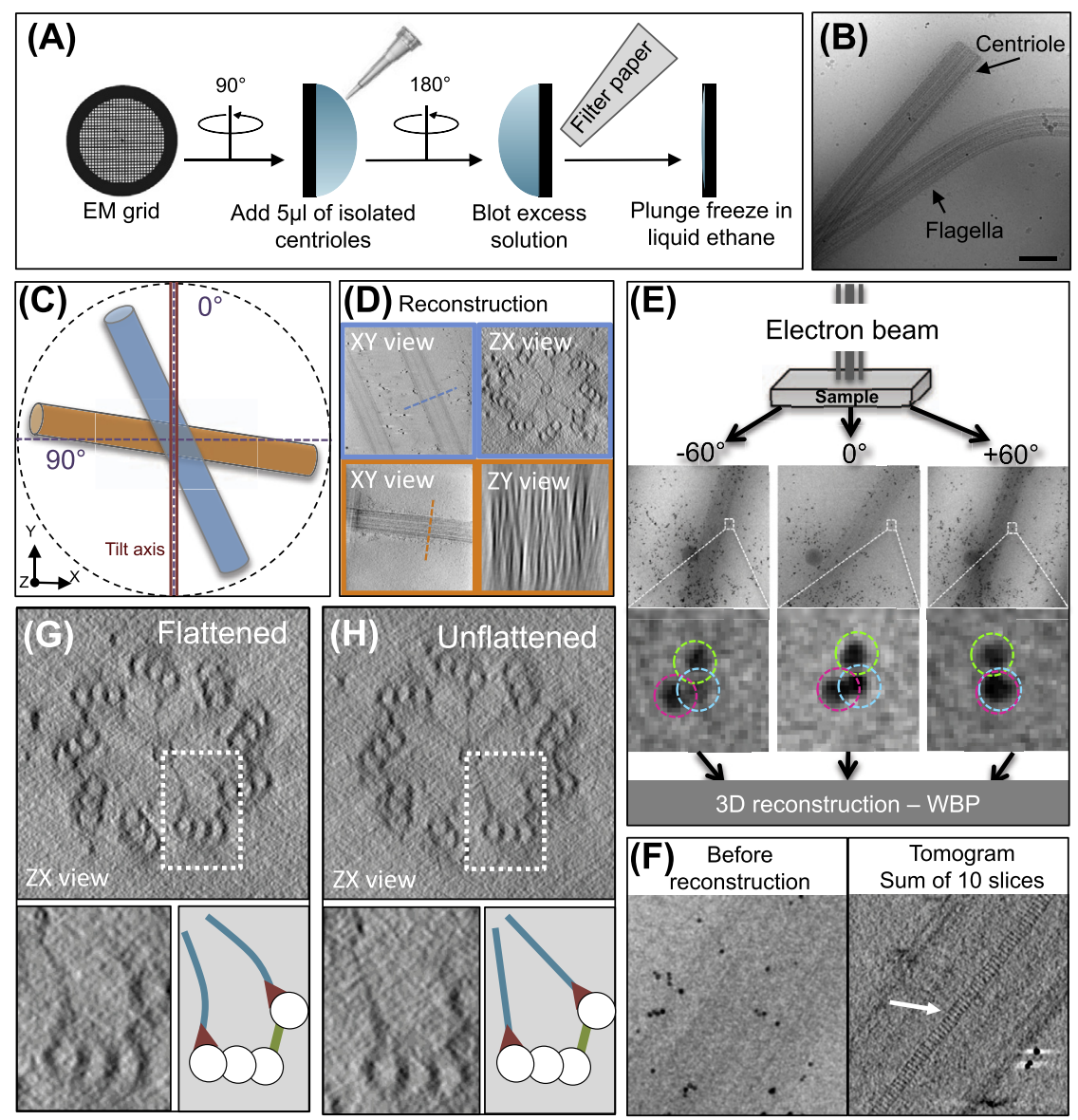

FIGURE 3 Cryotomography of isolated centrioles.

(A) Preparation of EM grids for cryomicroscopy. (B) Image of a centriole and a cilium in cryomicroscopy. Scale bar: $200 \mathrm{~nm}$. (C) Sample orientation compared to the tilt axis (red (dark gray in print versions)). In blue (light gray in print versions), the sample is almost parallel to the tilt axis and in orange (gray in print versions), perpendicular to it. (D) Effects of the missing wedge depending on sample orientation. The sample in blue (light gray in print versions) exhibits a good reconstruction in the ZX view (blue (light gray in print versions)), whereas the sample in orange (gray in print versions) is not reconstructed correctly in the ZY view. (E) Tomogram acquisition and reconstruction: fiducial markers (here three gold beads are highlighted) can be followed at different tilts. The tomogram is reconstructed using a weighted back projection (WBP). (F) Before tomogram reconstruction $\left(0^{\circ}\right.$ tilt), structures inside the centriole are not visible, but they appear after tomogram reconstruction (Tomogram), as exemplified by the central hub of the cartwheel (White arrow (dark gray in print versions)). ( $G$ and $H$ ) ZX views of tomogram reconstructions exemplifying a flattened tomogram with bent radial spokes $(G)$ or unflattened with characteristic straight radial spokes $(H)$. 
tomography. This concentration should be calibrated before use by analyzing gold beads on their own at different dilutions by cryo-EM.

- Use L5 style tweezers with a clamping ring to catch the extremity of a cryo-EM carbon film grid (Lacey 300 microMesh, EMS) (Figure 3(A)).

- Deposit $5 \mu \mathrm{L}$ of the purified centrioles on the surface of the cryo-EM grid using a $20-\mu \mathrm{L}$ tip cut at the extremity to have a larger diameter and thus preserve the structure of the long centriole/flagella complex (Figure 3(A)).

- Turn the grid by $180^{\circ}$. Remove the excess liquid by blotting the surface of the grid using a Whatman $\mathrm{n}^{\circ} 4$ filter paper in order to form a thin layer of liquid (approximately 50-300 nm, this cannot be controlled precisely). Immediately after blotting, plunge the grid with the tweezers into liquid ethane cooled at the temperature of liquid nitrogen $\left(-180{ }^{\circ} \mathrm{C}\right.$ ) to vitrify the sample (Figure $3(\mathrm{~A})$ ).

Note: To perform this vitrification step, a homemade plunging system can be used; alternatively, use a commercial setup such as a Vitrobot (FEI) or the Leica EM GP system.

- Transfer the grid to an electron microscope cryoholder. Ensure that the grid always stays in liquid nitrogen (or in nitrogen gas above the liquid nitrogen) during the transfer.

- Introduce the cryoholder containing the grid into the cryomicroscope. For optimal imaging, it is preferable to use a microscope operating at $200 \mathrm{keV}$ and equipped with an FEG. The microscope should be cooled at the temperature of liquid nitrogen before use.

- Select a zone in the grid containing a centriole, which is easily distinguished from a flagellum owing to its shape, centrioles being straight, whereas flagella are usually bent (Figure 3(B)). The observation should be conducted at very low magnification (i.e., $6000 \times$ ) but with a strong defocus (around $100 \mu \mathrm{m}$ ) in order to see the organelle in the vitreous ice (Figure 3(B)).

Note: After detection of a centriole at low magnification, ensure that the orientation of the centriole axis is close to parallel to the tilt axis of the microscope; this will be critical for the quality of the reconstruction due to the "missing wedge" effect. As exemplified in Figure 3(C)-(D), if the sample axis is close to the tilt axis (blue cylinder), the reconstruction will be suitable for further analysis by subtomogram averaging. In contrast, if the sample axis is close to $90^{\circ}$ compared with the tilt axis (orange cylinder), a strong artifact will be present in the tomogram reconstruction, precluding further analysis. For more details, regarding the missing wedge effect, see Guesdon, Blestel, Kervrann, and Chrétien (2013).

\subsubsection{Tomogram acquisition}

- After selection of a centriole at low magnification, use the low dose mode to acquire an image at high magnification in a region close to the centriole. Set the defocus at $\sim 2-4 \mu \mathrm{m}$. Note that this calibration step should not be conducted on 
the centriole itself, the structure of which would be affected by the electron beam (Baker \& Rubinstein, 2010).

- Perform the acquisition of the tomogram. Images are recorded at $29,000 \times$ magnification and a final pixel size of $0.748 \mathrm{~nm}$. Note that images can be collected using a $2 \mathrm{k}$ charge-coupled device (CCD) camera, but a $4 \mathrm{k}$ camera is preferable due to the possibility of using binning 2 during acquisition, thus reducing sample degradation by the electron beam.

- Acquire the tilt series covering an angular range from $-60^{\circ}$ to $+60^{\circ}$ with $2^{\circ}$ increments.

Note: Acquisition parameters (binning, number of images, angle increment) can be changed, but the final dose of electrons per surface area should not exceed $70 \mathrm{e}^{-} /$ $\AA^{2}$ in order to preserve the integrity of the sample and thus the final resolution (Kudryashev, Castaño-Díez, \& Stahlberg, 2012).

\subsubsection{Tomogram reconstruction using IMOD}

To start the tomogram reconstruction using IMOD, a stack of image files containing all images of the tomogram acquisition and the parameters used should be created in an .mrc format. The FEI software generates this file automatically, but the JEOL software does not. To generate an .mrc file in this case, an ImageJ plugin can be used to transform a stack with .tif format to an .mrc format. This plugin is mentioned in the Material and reagent section for the tomogram reconstruction software.

Note: This part will not explain in detail how to use the software etomo (IMOD package, Boulder, Colorado, (Kremer, Mastronarde, \& McIntosh, 1996)). For a detailed tutorial, please use this link:

http://bio3d.colorado.edu/imod/doc/etomoTutorial.html.

- Rename the extension ".mrc" of the stack to ".st," which indicates to the software that the tomogram is a single tilt acquisition.

- Load the tomogram and the angles file in etomo.

- Start the alignment of images using the fiducial mode in etomo. Use the 10-nm gold fiducial markers to precisely align the images (Figure 3(E)).

- Carefully verify that the software tracked the beads correctly in all tilt-angle images. As exemplified in Figure 3(E), beads can overlap sometimes at different angles (see colored dashed lines monitoring three gold beads in the Figure 3(E)).

- After correct alignment, reconstruct the tomogram using R-weighted back projection in etomo. As shown in Figure 3(F), after reconstruction internal structures such as the central hub (red arrow) can be visualized.

\subsection{SUBTOMOGRAM AVERAGING (FIGURE 4)}

In tomography, the final $3 \mathrm{D}$ reconstruction is known to be imperfect due to the missing wedge effect. This is due to the impossibility of acquiring images at high 
204 CHAPTER 11 Isolation, cryotomography, and 3D reconstruction of centrioles
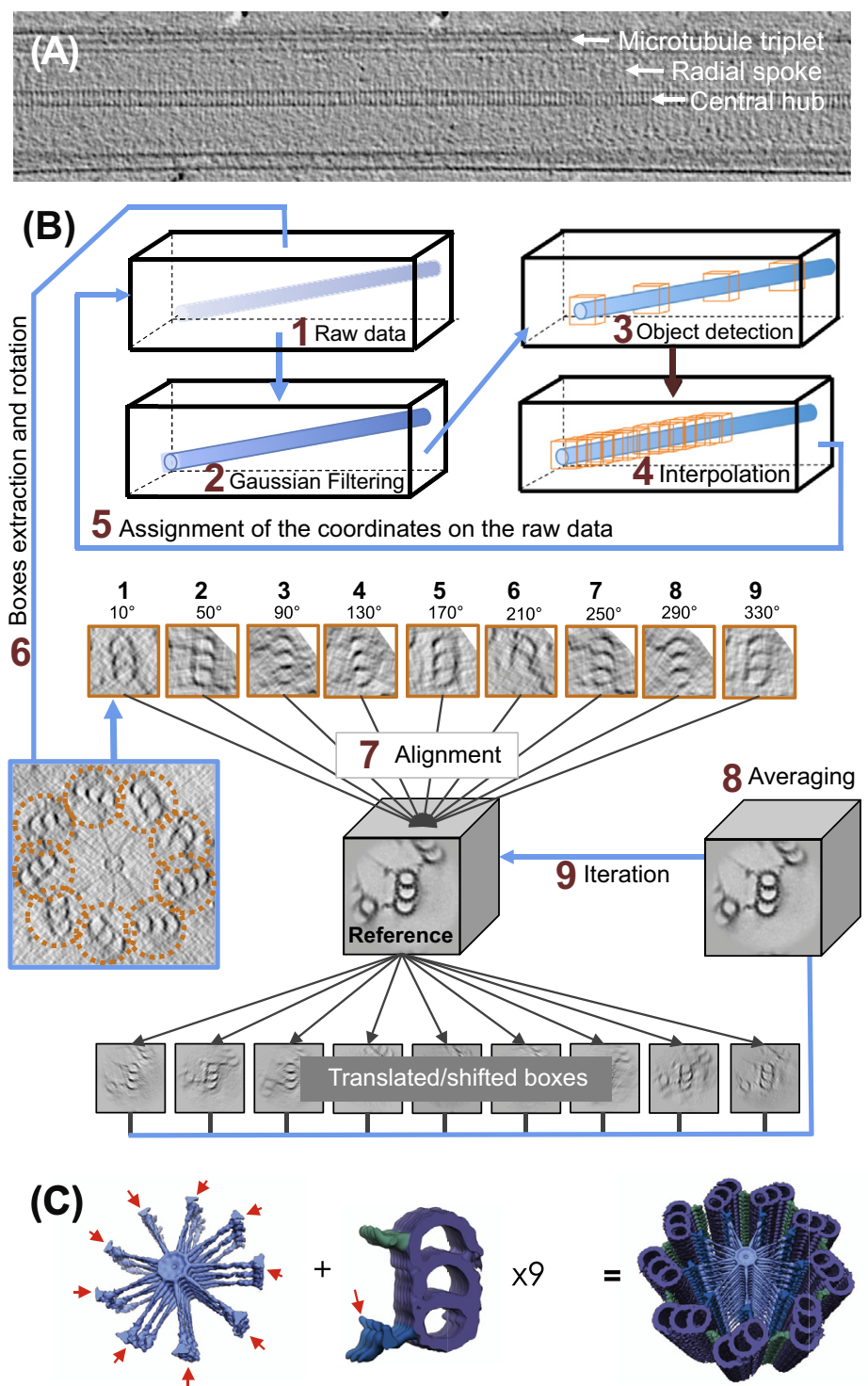

FIGURE 4 Subtomogram averaging.

(A) Cryotomogram section of a Trichonympha centriole. Microtubule triplets, the central hub of the cartwheel, and the radial spokes are highlighted. The radial spokes are difficult to visualize due to their low density. (B) Steps of image processing. The tomogram is first duplicated to preserve a version of the raw data (1) and then filtered using a Gaussian Filter (2). Detection of a specific microtubule triplet is done by picking five different regions along the structure (3). Those coordinates are saved and used to calculate a curve that corresponds to the trajectory of the microtubule triplet. This curve is used to calculate new 
angles, limiting the information during tomogram reconstruction and resulting in low information in the z-axis. The missing wedge effect is compensated in the case of the centriole by taking advantage of the ninefold symmetry of the structure during subtomogram averaging (Bui \& Ishikawa, 2013; Guichard et al., 2013; Li et al., 2011).

To perform subtomogram averaging, it is crucial to use tomograms of centrioles that have not been compressed. Such compression can appear during sample preparation and especially during formation of the thin ice layer before freezing. If the ice layer is thinner than the sample (i.e., less than $\sim 250 \mathrm{~nm}$ ), the centrioles will be affected. Moreover, the centriole can be compressed by touching the carbon edges of the cryo-EM grid. Sometimes the compression is not visible before reconstruction. As exemplified in Figure 3(G) and $(\mathrm{H})$, compression can affect only a subset of the centrioles, which are probably less rigid than others (blue, radial spokes of the cartwheel). In the case of structural analysis of the radial spokes for instance, it is very important to find centrioles without compression to avoid problem of averaging by merging straight radial spokes and abnormal curved radial spokes.

Figure 4(A) shows a cryoelectron tomogram with the different regions of the centriole, whereas Figure 4(B) illustrates the steps applied to the raw data to obtain a 3D structure: Duplication of the tomogram stack to preserve a version of the raw data (1), Gaussian filtering (2), object detection (3), interpolation (4), assignment of the coordinates on the raw tomogram (5), box extraction (6), alignment (7), and averaging (8). The steps of alignment and averaging are an iterative procedure (9).

- Due to the low contrast and high noise, the reconstructed tomogram is first duplicated to keep the initial raw volume and then filtered using a Gaussian filter (or a low-pass filter) in order to help visualization of the centriolar microtubules in the tomogram (Figure 4(B), steps 1 and 2). This step can be done using ImageJ, Bsoft, or Spider softwares.

- Using Bsoft in a Bshow mode, continuous microtubule triplets are detected in the tomogram and picked (micrograph/pick particles) in five different regions along the microtubule blade (Figure 4(B), step 3). Then the coordinates are saved using the item "micrograph/write parameters" in Bshow. This step creates a .star file. This step is repeated for each microtubule blade.

Note: The picked boxes along the blade are used as landmarks to calculate a curve that simulates the trajectory of the microtubule triplets. This curve is generated

coordinates spaced by $17 \mathrm{~nm}$ (4). Using these new coordinates, subtomograms are extracted from the raw tomogram (5). This step is repeated for the nine microtubule triplets (6). After extraction, boxes from each triplet are rotated to be in the same orientation as the reference map and processed to align them with the reference (7). The resulting translated boxes are used for the averaging (8), and the output is used as a new reference iteratively (9). (C) $3 \mathrm{D}$ reconstruction views of the cartwheel and the microtubule triplet. These two regions have been reconstructed separately. Using common features between the two maps (i.e., the pinhead structure, red (dark gray in print versions) arrows) and by duplicating 9 times the triplet map, the complete architecture of the centriole proximal region is reconstructed. 
by interpolating the spline passing through the points, applying iteratively the centripetal scheme described in Lee (1989). After curve determination, 3D coordinates of $17 \mathrm{~nm}$-spaced boxes are calculated along the microtubules. We have written a Matlab $^{\circledR}$ script, freely available on our Web site (http://gonczy-lab.epfl.ch/ resources), to perform this step. Using the .star file with manually selected coordinates as an input, the script generates a new .star file (called filename I.STAR) with the coordinates of 17-nm-spaced boxes along the interpolated cubic spline curve.

- Use the coordinates saved previously for each .star file to generate the new interpolated coordinates of 17-nm-spaced boxes for each selected microtubule blade (Figure 4(A) and (B) step 4).

- Using the command-line mode of Bsoft (Heymann, Cardone, Winkler, \& Steven, 2008), extract the subtomogram based on the new coordinates file using the command "bpick" on the raw tomogram (Figure 4(B), steps 5 and 6).

- Because of the ninefold symmetry, each microtubule triplet should be rotated with an increment angle of $40^{\circ}$, from the first microtubule blade to the last microtubule blade (Figure 4(B), step 6).

- Align all extracted and rotated subtomograms using PEET (Nicastro et al., 2006), Jsubtomo (Huiskonen et al., 2010) or Dynamo (Castaño-Díez, Kudryashev, Arheit, \& Stahlberg, 2012). As for IMOD, theses pieces of software are very well described, and tutorials are available elsewhere (Figure 4(B), steps 7 and 8).

To perform this alignment, a reference map is needed. Three maps are available for centriole in the Electron microscopy Data Bank:

- Chlamydomonas basal body microtubule triplet: EMD-5252 (Li et al., 2011)

- Trichonympha basal body microtubule triplet: EMD-2330 (Guichard et al., 2013)

- Trichonympha cartwheel: EMD-2329 (Guichard et al., 2013)

- After alignment, the new averaged map can be visualized using UCSF Chimera (Pettersen et al., 2004).

\section{CONCLUSIONS}

We presented a step-by-step protocol to purify and analyze the proximal part of Trichonympha centrioles in their native state with unprecedented detail using cryotomography (Figure 4(C)). This method can be applied more generally to study centrioles from other species. By taking advantage of repetitive structures, it is possible to use such periodicities to extract multiple copies of the same structural unit to perform subtomogram averaging. However, this method requires a large number of copies to obtain a robust 3D reconstruction (2000-5000 copies, (Guichard et al., 2012, 2013; Li et al., 2011)). This represents a prohibitive limitation for small or nonperiodical structures. For instance, the canonical height of the 
cartwheel is $\sim 100 \mathrm{~nm}$, with about 5 repeats, meaning that only 5 copies can be extracted per centriole (using the ninefold symmetry, these 5 copies can be turned into 45 final copies.). To circumvent this limitation, we took advantage of the exceptionally long proximal region of the Trichonympha centriole, which enabled us to extract about 900 copies per tomogram. Using only 6 tomograms, we could thus obtain the $\sim 5000$ copies needed for correct subtomogram averaging. For the analysis of a small or nonperiodic structure, the strategy would then be to acquire a much larger number of tomograms and extract few boxes from each tomogram before averaging. For instance, in the case of the canonical cartwheel, 50-100 tomograms of unflattened and well-oriented centrioles should be acquired. Although this number of tomograms was difficult to reach until recently, software and hardware development that allow automatic tomogram acquisition open the possibility to perform this task. Moreover, the development of new camera detectors with better contrast and resolution (Grigorieff, 2013; Ruskin, Yu, \& Grigorieff, 2013) allows one to decrease the number of particles needed for subtomogram averaging. These advances will bring the architecture of centrioles from many species within reach and thus are expected to yield exciting new discoveries.

\section{ACKNOWLEDGMENTS}

We thank Veronika Nemcikova Villimova and Nicola J. Brown for reading the manuscript. We thank Capucine Hachet for help in preparing Figure 2(A). Work on centriole formation in the Gönczy laboratory is funded notably by the European Research Council (ERC) (AdG 233335 and AdG 340227). A.N. was supported by a SystemsX.ch Transition Postdoc Fellowship (SXFSI0_141995).

\section{REFERENCES}

Allen, R. D. (1969). The morphogenesis of basal bodies and accessory structures of the cortex of the ciliated protozoan Tetrahymena pyriformis. The Journal of Cell Biology, 40, 716-733.

Baker, L. A., \& Rubinstein, J. L. (2010). Radiation damage in electron cryomicroscopy. Elsevier Masson SAS.

Bornens, M., Paintrand, M., Berges, J., Marty, M. C., \& Karsenti, E. (1987). Structural and chemical characterization of isolated centrosomes. Cell Motility and the Cytoskeleton, 8, 238-249.

Bouchet-Marquis, C., \& Hoenger, A. (2011). Cryo-electron tomography on vitrified sections: a critical analysis of benefits and limitations for structural cell biology. Micron, $42,152-162$.

Bui, K. H., \& Ishikawa, T. (2013). 3D structural analysis of flagella/cilia by cryo-electron tomography (1st ed.). Elsevier Inc.

Castaño-Díez, D., Kudryashev, M., Arheit, M., \& Stahlberg, H. (2012). Dynamo: a flexible, user-friendly development tool for subtomogram averaging of cryo-EM data in highperformance computing environments. Journal of Structural Biology, 178, 139-151. 


\section{CHAPTER 11 Isolation, cryotomography, and 3D reconstruction of centrioles}

Cavalier-Smith, T. (1974). Basal body and flagellar development during the vegetative cell cycle and the sexual cycle of Chlamydomonas reinhardtii. Journal of Cell Science, $16,529$.

Dippell, R. V. (1968). The development of basal bodies in paramecium. Proceedings of the National Academy of Sciences of the United States of America, 61, 461.

Dubochet, J. (2012). Cryo-EM-the first thirty years. Journal of Microscopy, 245, 221-224.

Dubochet, J., \& McDowall, A. W. (1981). Vitrification of pure water for electron microscopy. Journal of Microscopy, 124, RP3-RP4.

Dubochet, J., \& Sartori Blanc, N. (2001). The cell in absence of aggregation artifacts. Micron (Oxford, England: 1993), 32, 91-99.

Geimer, S., \& Melkonian, M. (2004). The ultrastructure of the Chlamydomonas reinhardtii basal apparatus: identification of an early marker of radial asymmetry inherent in the basal body. Journal of Cell Science, 117, 2663-2674.

Gönczy, P. (2012). Towards a molecular architecture of centriole assembly. Nature Reviews Molecular Cell Biology, 13, 425-435.

Grassucci, R. A., Taylor, D. J., \& Frank, J. (2007). Preparation of macromolecular complexes for cryo-electron microscopy. Nature Protocols, 2, 3239-3246.

Grigorieff, N. (2013). Direct detection pays off for electron cryo-microscopy. eLife, 2013, 2-4.

Guesdon, A., Blestel, S., Kervrann, C., \& Chrétien, D. (2013). Single versus dual-axis cryoelectron tomography of microtubules assembled in vitro: limits and perspectives. Journal of Structural Biology, 181, 169-178.

Guichard, P., Chrétien, D., Marco, S., \& Tassin, A.-M. (2010). Procentriole assembly revealed by cryo-electron tomography. EMBO Journal, 29, 1565-1572.

Guichard, P., Desfosses, A., Maheshwari, A., Hachet, V., Dietrich, C., Brune, A., et al. (2012). Cartwheel architecture of Trichonympha basal body. Science, 337, 2012.

Guichard, P., Hachet, V., Majubu, N., Neves, A., Demurtas, D., Olieric, N., et al. (2013). Native architecture of the centriole proximal region reveals features underlying its 9-fold radial symmetry. Current Biology: CB, 23, 1620-1628.

Heymann, J. B., Cardone, G., Winkler, D. C., \& Steven, A. C. (2008). Computational resources for cryo-electron tomography in Bsoft. Journal of Structural Biology, 161, $232-242$.

Huiskonen, J. T., Hepojoki, J., Laurinmäki, P., Vaheri, A., Lankinen, H., Butcher, S. J., et al. (2010). Electron cryotomography of Tula hantavirus suggests a unique assembly paradigm for enveloped viruses. Journal of Virology, 84, 4889-4897.

Kremer, J. R., Mastronarde, D. N., \& McIntosh, J. R. (1996). Computer visualization of threedimensional image data using IMOD. Journal of Structural Biology, 116, 71-76.

Kudryashev, M., Castaño-Díez, D., \& Stahlberg, H. (2012). Limiting factors in single particle cryo electron tomography. Computational and Structural Biotechnology Journal, 1 .

Lee, E. T. Y. (1989). Choosing nodes in parametric curve interpolation. Computer-Aided Design, 21, 363-370.

Li, S., Fernandez, J.-J., Marshall, W. F., \& Agard, D. A. (2011). Three-dimensional structure of basal body triplet revealed by electron cryo-tomography. EMBO Journal, 1-11.

Nicastro, D., Schwartz, C., Pierson, J., Gaudette, R., Porter, M. E., \& McIntosh, J. R. (2006). The molecular architecture of axonemes revealed by cryoelectron tomography. Science (New York, N.Y.), 313, 944-948.

Nigg, E. A., \& Raff, J. W. (2009). Centrioles, centrosomes, and cilia in health and disease. Cell, 139, 663-678. 
Pettersen, E. F., Goddard, T. D., Huang, C. C., Couch, G. S., Greenblatt, D. M., Meng, E. C., et al. (2004). UCSF Chimera-a visualization system for exploratory research and analysis. Journal of Computational Chemistry, 25, 1605-1612.

Ruskin, R. S., Yu, Z., \& Grigorieff, N. (2013). Quantitative characterization of electron detectors for transmission electron microscopy. Journal of Structural Biology, 184, 385-393.

Schmidt, H. (1956). Studies on Flagellata in intestine of termites. II. Wood particles as cell inclusions and nutrition in Trichonympha and Joenia. Zeitschrift fur Parasitenkunde (Berlin, Germany), 17, 269-275.

Vorobjev, I. A., \& Chentsov, Yu S. (1982). Centrioles in the cell cycle. I. Epithelial cells. Journal of Cell Biology, 93, 938-949. 